\title{
Simulation Studies of a "Nanogun" Based on Carbon Nanotubes
}

\author{
Yitao Dai, Chun Tang, and Wanlin Guo $(\bowtie)$ \\ Institute of Nano Science, Nanjing University of Aeronautics and Astronautics, Nanjing 210016, China \\ Received: 3 May 2008/ Revised: 30 June 2008/Accepted: 30 June 2008 \\ (O)Tsinghua Press and Springer-Verlag 2008
}

\begin{abstract}
Quantum mechanical molecular dynamics simulations show that electrically neutral carbon nanotubes or fullerene balls housed in an outer carbon nanotube can be driven into motion by charging the outer tube uniformly. Positively and negatively charged outer tube are found to have quite different actions on the initially neutral nanotubes or fullerene balls. A positively charged tube can drive out the molecule inside it out at speeds over $1 \mathrm{~km} / \mathrm{s}$, just like a "nanogun", while a negatively charged tube can drive the molecule into oscillation inside it and can absorb inwards a neutral molecule in the vicinity of its open end, like a "nanomanipulator". The results demonstrate that changing the charge environment in specific ways may open the door to conceptually new nano/molecular electromechanical devices.
\end{abstract}

\section{KEYWORDS}

Energy conversion, carbon nanotube, neutral molecule, driving mechanisms

\section{Introduction}

The ability to drive or power functional molecular devices based on unconventional operating principles is of essential interest in various scientific disciplines and technologies [1-5]. A large variety of driving mechanisms have been found by using electromagnetic, electrostatic, or domain-switching principles for devices containing a net charge [6], magnetic moment [7] or electrical domains [8]. Based on such principles, external magnetic or electric fields have been used to provide the driving force. However, neutral molecules have no net charge, magnetic moment or electrical domain, and hence finding a way of driving them remains a challenge $[9,10]$. In contrast to artificial systems, biomolecular systems such as protein-motors [11, 12], voltagegated ion channels [13], and ATP-triggered guest releasers [14] are surprisingly efficient and can work in response to very slight changes in charge [15] or other factors [16].

When size is reduced to the nano or molecular scale, the local fields of matter or structure play an important role, comparable to external fields, and novel driving mechanisms may become dominant. Carbon nanotubes (CNTs) are well known for their exceptional mechanical and electronic properties [17], and recent research has also shown that CNTs and fullerenes are very sensitive to external charge environments or applied fields as the $\mathrm{sp}^{2}$ carbon atoms have a rich $\pi$-electron cloud $[18,19]$. High quality multiwalled and biwalled CNTs and CNT structures containing fullerene balls or bamboo sections can be easily fabricated [20,21]. These carbon structures can be independent and free to move inside a housing nanotube with ultra-low friction [22-

Address correspondence to wlguo@nuaa.edu.cn 
24]. The outer tube can be engineered to be open one or both ends, be separate or in bulk films, and also can be fixed to an electrode [24]. Without an applied charge or external field, the core tube or fullerene will remain held in the housing tube by virtue of van der Waals interactions [22].

Here we show by extensive quantum mechanical molecular dynamics simulations on an ideal model that an electrically neutral fullerene ball or carbon nanotube inside an outer housing carbon nanotube can be driven to move when the housing tube is uniformly charged. A positive charge on the house can drive out the neutral core tube or fullerene ball at high speed like a bullet, while a negative charge can drive it to oscillate within the housing tube.

\section{Modelling and methods}

The typical model considered in this work is a short capped $(5,5)$ CNT core or a $\mathrm{C}_{60}$ molecule in an outer housing $(18,0)$ CNT with one end capped and the other open. The initial system is firstly optimized by the AMBER molecular mechanics force field, the outer part is then fixed and an energy minimization is performed using the parametrization 3 of the modified neglect of diatomic orbital (PM3) quantum mechanics (QM) method for the inner part. The $\mathrm{MM}+$ molecular mechanics force field (the extension of version 2 of Allinger's molecular mechanics force field) can also be used for the energy minimization of the initial system and no essential difference was found in the subsequent QM simulations. Finally, the outer housing tube is charged with a specific uniform density and the core part is calculated using the QM method to provide the driving force on each atom. Once the energy parameters of the atoms are obtained at one time step, the motion parameters for each atom in the next time step can be determined by Newton' $s$ laws of motion and the velocity and acceleration of the center of mass of the core can be obtained. All the QM molecular dynamics (QMMD) simulations were performed using a Verlet algorithm with a time step of $1 \mathrm{fs}$ at zero initial temperature within a constant energy assumption. The total energies of the whole system are guaranteed to converge to $10^{-5} \mathrm{kcal} / \mathrm{mol}$ for all the QM calculations.
In the QMMD simulations, the positions and velocities of the atoms in the system are determined by Newton's laws of motion, while the interactions between the atoms are calculated by solving the Schrödinger equation

$$
H \Psi=E \Psi
$$

where $H$ is the Hamiltonian operator, $E$ is the total energy of the system, and $\Psi$ is the system wavefunction. The Schrödinger equation is difficult to solve exactly for large molecular systems; however, it is possible to solve the equation in accordance with the Born-Oppenheimer approximation that the motion of the electrons is decoupled from the motion of the nuclei and the Hartree-Fock approximation that a many-electron problem is translated into a single electron problem. Thus,

$$
H_{i} \Psi_{i}=\varepsilon_{i} \Psi_{i}
$$

where $H_{i}$ is an effective one-electron Hamiltonian, $\Psi_{i}$ is a molecular orbital, and $\varepsilon_{i}$ is the orbital energy of an electron in the orbital $\Psi_{i}$. When the linear combination of atomic orbitals (LCAO) assumption is used, $\Psi_{i}=\sum_{\mu} C_{\mu i} \phi_{\mu}$ in which $\phi_{\mu}$ is the $\mu$ th atomic orbital and $C_{\mu i}$ is the coefficient. The Hartree-Fock $\left(\mathrm{H}^{-}\right.$ F) equation can be conveniently written as a matrix form, or the Roothaan-Hall equation

$$
F C=S C E
$$

with the Fock matrix $F$ in the form

$$
\begin{aligned}
F_{\mu v}= & \int \mathrm{d} v \phi_{\mu}\left[-\frac{1}{2} \nabla_{i}^{2}-\sum_{A=1}^{M} \frac{Z_{A}}{r_{i A}}\right] \phi_{v} \\
& +\sum_{\lambda=1}^{K} \sum_{\sigma=1}^{K} P_{\lambda \sigma}\left[(\mu v \mid \lambda \sigma)-\frac{1}{2}(\mu \lambda \mid v \sigma)\right]+V_{\mu v}
\end{aligned}
$$

where the first term is the core energy, and the second term is the energy arising from the Coulomb and exchange interactions, $\boldsymbol{P}$ is the charge density matrix and $P_{\lambda \sigma}=2 \sum_{i=1}^{N / 2} C_{\lambda i} C_{\sigma i}, V_{\mu \nu}$ represents the influence of external fields, $S$ is the overlap integrals matrix and $S_{\mu v}=\int \mathrm{d} v \phi_{\mu} \phi_{v}, C$ is the coefficient matrix, and $E$ is the orbital energy diagonal matrix. $(\mu v \mid \lambda \sigma)$ and $(\mu \lambda \mid$ $v \sigma)$ are two-electron integrals that may involve up to four different basis functions $\left(\phi_{\mu}^{\mathrm{A}}, \phi_{v}^{\mathrm{B}}, \phi_{\lambda}^{\mathrm{C}}, \phi_{\sigma}^{\mathrm{D}}\right)$, which may in turn be located at four different centers.

As the whole system contains a large number of atoms and the interaction between the "bullet" and the outer tube housing at the van der Waals distance cannot be described properly by either density

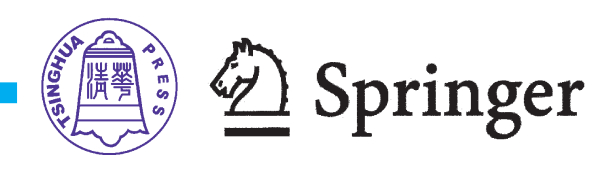


functional theory (DFT) or the Hartree-Fock method [25], a special method called the Mixed Model is used to describe the "nanogun" system. In this method, the outer tube is treated by a classical mechanical model, where the positions and charges of the atoms are fixed and the Slater exponents of $\mathrm{s}$ orbitals are used for all the charges; the core part is described by the semi-empirical quantum mechanics method PM3 $[26,27]$, which considers only the valence electrons of the system and neglects the three- or four-center integrals in the Fock matrix. The interaction between the charges and nuclei of the "bullet" and the housing are considered using the core Hamiltonian correction between the quantum mechanical "bullet" and the classical outer tube housing as follows

$$
\Delta H_{\mu v}=-\sum_{\mathrm{B}}\left(Z_{\mathrm{B}}-Q_{\mathrm{B}}\right)\left(\mu_{\mathrm{A}} v_{\mathrm{A}} \mid s_{\mathrm{B}} s_{\mathrm{B}}\right)
$$

The interaction energy between the nuclei of the atoms of the "bullet" and all the nuclear and electronic charges on the atoms of the housing is

$$
\begin{aligned}
\Delta E= & \sum_{(\mathrm{A}, \mathrm{B})}\left\{Z _ { \mathrm { A } } Z _ { \mathrm { B } } ( s _ { \mathrm { A } } s _ { \mathrm { A } } | s _ { \mathrm { B } } s _ { \mathrm { B } } ) \left[1+\exp \left(-\alpha_{\mathrm{A}} R_{\mathrm{AB}}\right)\right.\right. \\
& \left.\left.+\frac{\exp \left(-\alpha_{\mathrm{A}} R_{\mathrm{AB}}\right)}{R_{\mathrm{AB}}}\right]-Z_{\mathrm{A}} Q_{\mathrm{B}}\left(s_{\mathrm{A}} s_{\mathrm{A}} \mid s_{\mathrm{B}} s_{\mathrm{B}}\right)\right\}
\end{aligned}
$$

with

$$
\left(s_{\mathrm{A}} s_{\mathrm{A}} \mid s_{\mathrm{B}} s_{\mathrm{B}}\right)=\left[R_{\mathrm{AB}}^{2} \frac{1}{2}\left(\frac{1}{\mathrm{AM}_{\mathrm{A}}}+\frac{1}{\mathrm{AM}_{\mathrm{B}}}\right)\right]^{-1 / 2}
$$

where the subscript A represents atoms on the "bullet" and B represents atoms on the housing, $s$ is the s-type orbital, $R_{\mathrm{AB}}$ is the distance between atom $\mathrm{A}$ and atom $\mathrm{B}$, and AM are the monopole-monopole interaction parameters. $Z$ is the nuclear charge, $Q$ is the electronic charge, and $\alpha_{\mathrm{A}}$ is a monatomic parameter of atom A.

To check the validity of the PM3-based Mixed Model, both $\mathrm{H}-\mathrm{F}$ ab initio and Kohn-Sham DFT single point calculations were performed on the "nanogun" model used in the PM3 molecular dynamics simulations. Comparison of the calculated dipole moments using the three methods for a $\mathrm{C}_{60}$ fullerene "bullet" within a $(18,0)$ carbon nanotube housing charged with uniform distributed positive charge density of $+0.001 \mathrm{e} /$ atom is presented in Fig. 1(a). It can be seen that the variations in dipole moment with position of the "bullet" in the housing show the same trend for all three methods. The curves obtained using the $\mathrm{H}-\mathrm{F}$ ab initio and the DFT methods are almost the same. The values obtained using the PM3 method are slightly higher, but agree quite well with those of the $\mathrm{H}-\mathrm{F}$ ab initio and DFT methods. The PM3-based Mixed Model has also been shown to be valid for calculation of the interaction energy. As shown in Fig. 1(b), the variation in the interaction energy of two parallel benzene molecules as a function of separation was calculated and the results compared with those in the literature [28]. The Mixed Model predicts a similar trend to that of the $\operatorname{CCSD}(\mathrm{T})$ method, which is thought to be one of the most precise QM methods.

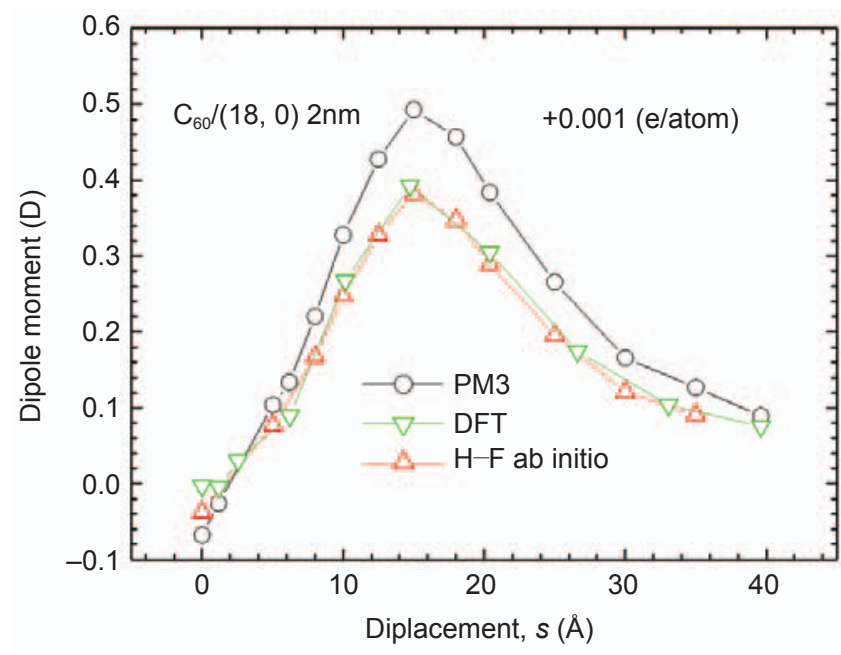

(a)

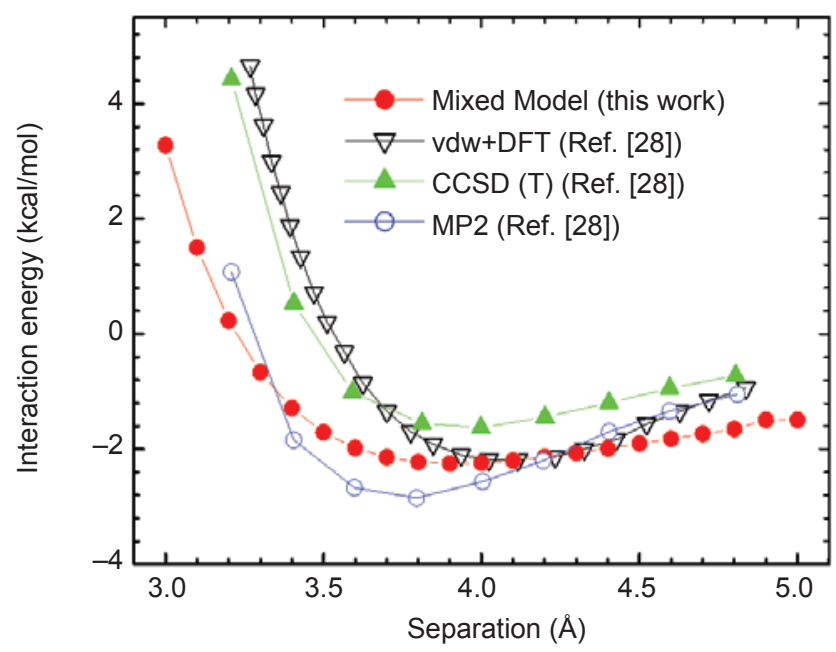

(b)

Figure 1 (a) Variation of the dipole moment of the $C_{60}$ "bullet" with its position in the outer $(18,0)$ tube housing with length of about $2.0 \mathrm{~nm}$. (b) Comparison of the interaction energy between two parallel benzene molecules obtained by the Mixed Model with the results in Ref. [28] 
The interaction energy and the dipole moment are directly related to the movement of the core part. The agreement between the interaction energies and the dipole moments with the results obtained by other methods with much higher precision demonstrates that the semiempirical Mixed Model is valid for QMMD simulations of "nano-gun" systems, at least for the models used in this work.

\section{Results and discussion}

When we charged the housing tube with a positive charge, our QMMD numerical calculations suggested that the encapsulated neutral core tube or fullerene ball can be driven out. A typical total energy curve of a neutral $\mathrm{C}_{60}$ molecule moving in a positively charged $(18,0)$ housing tube with a uniform charge density of $0.001 \mathrm{e} /$ atom is presented in Fig. 2. It can be seen that there are two sharp drops in energy when the fullerene ball leaves the bottom and the open end of the housing tube, while in the middle part of the housing, the energy remains relatively stable. This means that there are two acceleration stages corresponding to the two energy drops which force the ball out. Similar energy curves can be obtained for a CNT core in the charged housing.

Snapshots every 0.5 ps of the driving processes obtained by the QMMD simulations on three typical

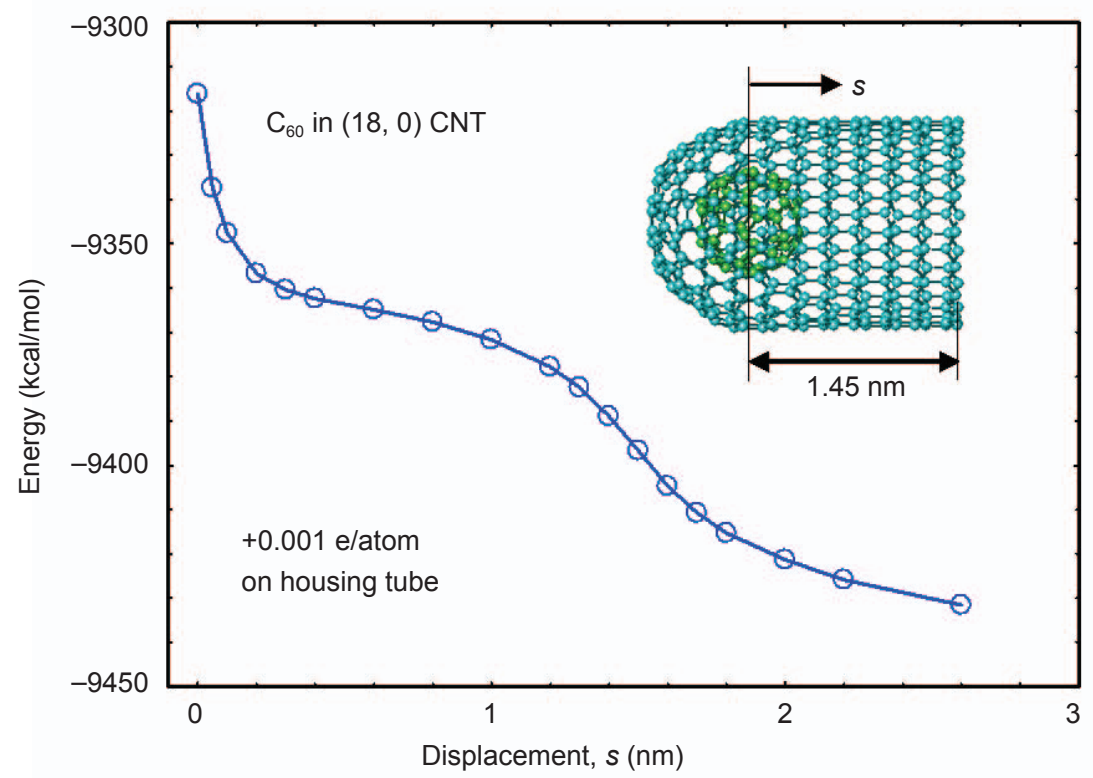

Figure 2 Variation in the energy of the neutral $C_{60}$ ball with its displacement from the bottom of the positively charged $(18,0)$ CNT housing systems of neutral CNTs or fullerene in charged housing CNTs are shown in Fig. 3(a) with time increasing from top to bottom. In all three cases, the outer tube housings are positively charged with uniform density of $+0.001 \mathrm{e} /$ atom. Case A shown in Fig. 3(a) shows a capped $(5,5)$ core tube with length about $2 \mathrm{~nm}$ shooting out from the open end of a fixed $(18,0)$ housing tube with length about $3.7 \mathrm{~nm}$. Case $\mathrm{B}$ in the same figure involves the same core tube in a shorter housing of about $2 \mathrm{~nm}$, and case $C$ involves a neutral $\mathrm{C}_{60}$ ball in the $2 \mathrm{~nm}$ housing. The speed of ejection of the neutral core in each case is over $1 \mathrm{~km} / \mathrm{s}$ as shown in Fig. 3(b). Thus, the system resembles a "nanogun" which drives the neutral "bullet" electrically. The velocity curve of the $(5,5) 2 \mathrm{~nm} /(18$, 0) $3.7 \mathrm{~nm}$ system (case A) contains four distinct regions: the acceleration region $\mathrm{I}$ is the initial stage with a displacement of less than about $0.3 \mathrm{~nm}$, with acceleration up to $2.6 \times 10^{15} \mathrm{~m} / \mathrm{s}^{2}$ and a corresponding driving force of $52 \mathrm{pN} /$ atom; in the following region II, the core tube moves in the housing with relatively stable speed, and when the core reaches the open end of the housing there comes the second acceleration region, region III, with an acceleration of $4.3 \times 10^{14} \mathrm{~m} / \mathrm{s}^{2}$ and driving force of $8.6 \mathrm{pN} /$ atom; when the core is completely out of the outer tube, region IV, the velocity reaches its maximum value of about $1540 \mathrm{~m} / \mathrm{s}$. The velocity curve of the $C_{60} /(18,0) 2 \mathrm{~nm}$ system (case C) has a similar shape with four regions, while the accelerations in the regions I and III are slightly higher with the maximum final velocity being 1340 $\mathrm{m} / \mathrm{s}$. The reason for the lower final velocity of $\mathrm{C}_{60}$ is that the acceleration region III is significantly shorter than that available to the 2-nm CNT core. When the outer tube is longer than the core, as in the cases A and C, the two acceleration stages are distinctly separated by a transition region II where the speed is relatively stable. However, when the neutral "bullet" is comparable in length to or longer than the housing, the transition region II disappears and the two acceleration stages merge, as shown in Fig. 3(b) for

\section{国然 Springer}




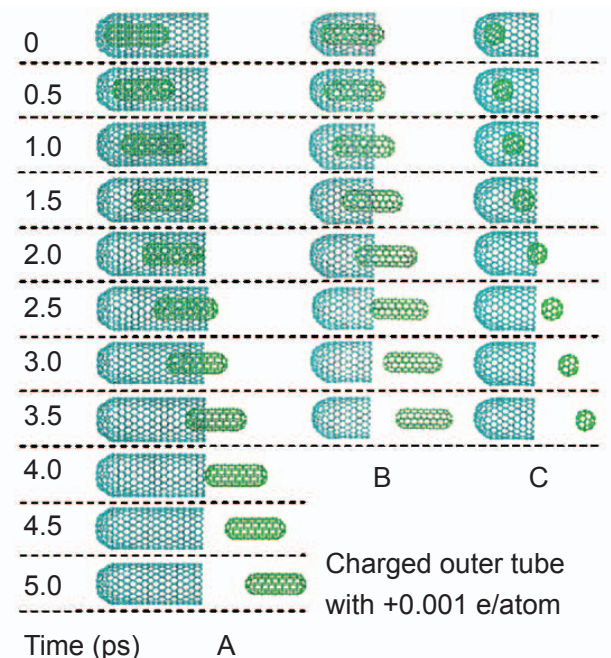

(a)

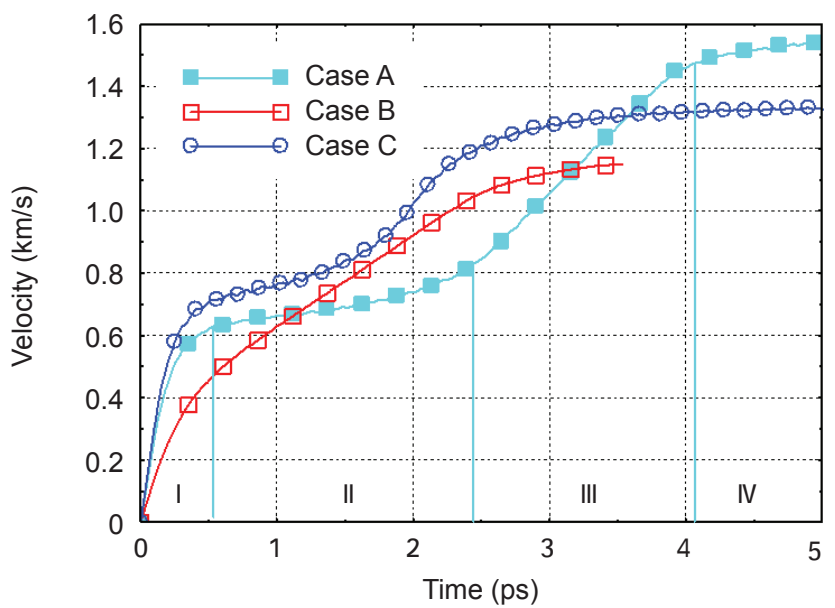

(b)

Figure 3 Neutral CNTs or fullerene "bullets" shooting out from positively charged CNT housings with speeds well over $1 \mathrm{~km} / \mathrm{s}$. (a) Snapshots of the neutral CNTs or fullerene ball driven out from the open end of the fixed CNT housing by its positive charge. With time increasing from top to bottom, the snapshot interval is $0.5 \mathrm{ps}$. The density of the charge on the housing is +0.001 e/atom and is evenly distributed. Case $\mathrm{A}$ : a capped $(5,5)$ tube containing 170 atoms with length of about $2.0 \mathrm{~nm}$ is shooting out from a fixed $(18,0)$ tube having 660 atoms and length of about $3.7 \mathrm{~nm}$ with one end open; Case B: a capped $(5,5)$ tube containing 170 atoms with length of about $2.0 \mathrm{~nm}$ is shooting out from the open end of a fixed $(18,0)$ tube having 372 atoms and length of about $2.0 \mathrm{~nm}$; Case $\mathrm{C}$ : a $\mathrm{C}_{60}$ fullerene ball is shooting out from the open end of a fixed $(18,0)$ tube having 372 atoms and length about $2.0 \mathrm{~nm}$. (b) The velocity curves of the "bullets" in the three cases

case B in which the "bullet" has the same length as the housing. The accelerations in the merged regions I and III are markedly lower, being $1.3 \times 10^{15}$ and $3.0 \times$ $10^{14} \mathrm{~m} / \mathrm{s}^{2}$, respectively. For each of the three cases, the accelerations in stages I and II of the order of $10^{15}$ and $10^{14} \mathrm{~m} / \mathrm{s}^{2}$, respectively correspond to driving forces on the neutral "bullet" of the order of 1-10 pN/atom, which is about 2 to 3 orders of magnitude higher than the friction force between the core and the housing which is typically $10^{-14} \mathrm{~N} /$ atom [22-24]. For a given charge density, the maximum velocity of the neutral "bullet" acquired in the two acceleration stages is dependent on the length of both the housing and bullet.

Increasing the density of the positive charge on the housing tube from +0.001 to $+0.005 \mathrm{e} /$ atom monotonically increases the speed of the neutral "bullet" in the $(5,5) 2 \mathrm{~nm} /(18,0) 2 \mathrm{~nm}$ "nanogun" (case B) from 1150 to about $2200 \mathrm{~m} / \mathrm{s}$ as shown in Fig. 4(a). Unlike the electrostatic operating principle used in many previous nanodevices [20, 24], the operating mechanism in the present systems is a quantum response of the neutral nanostructure of CNTs or fullerenes to the electrical environment created by the positively charged CNT house. Detailed quantum mechanical analyses show that the "bullet" changes its electronic structure and energy bands, and can be easily polarized in the charged housing; increasing the charge density on the house causes an increase in dipole moment in the "bullet" as shown in Fig. 4(b). For a given positive charge density, the dipole moment first increases and then decreases phase when the "bullet" leaves the housing. Therefore, the driving process is a quantum electrodynamic process. Further numerical calculations demonstrate that a positively charged housing tube with a charge density of $+0.001 \mathrm{e} /$ atom can shoot out more than one neutral "bullet" with speeds of over $1 \mathrm{~km} / \mathrm{s}$.

For a commensurate set-up of the zigzag housing and zigzag "bullet" system, a positively charged housing CNT can also shoot out the neutral "bullet" at speeds comparable to that of the incommensurate system, as shown in Fig. 4(c). Therefore, the chirality of the system has only a slight effect on the driving process.

Figure 5 shows the different effects of positively and negatively charged housing on a neutral $\mathrm{C}_{60}$ ball. It was unexpectedly found that only the positively charged housing tube can drive the ball out, while a negatively charged housing tube only drives the ball into periodic oscillating motion within it. The 


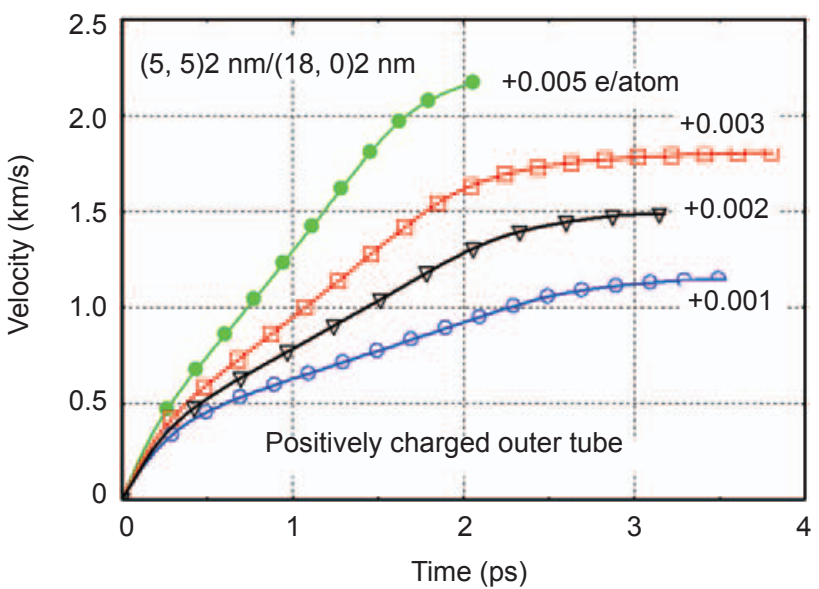

(a)

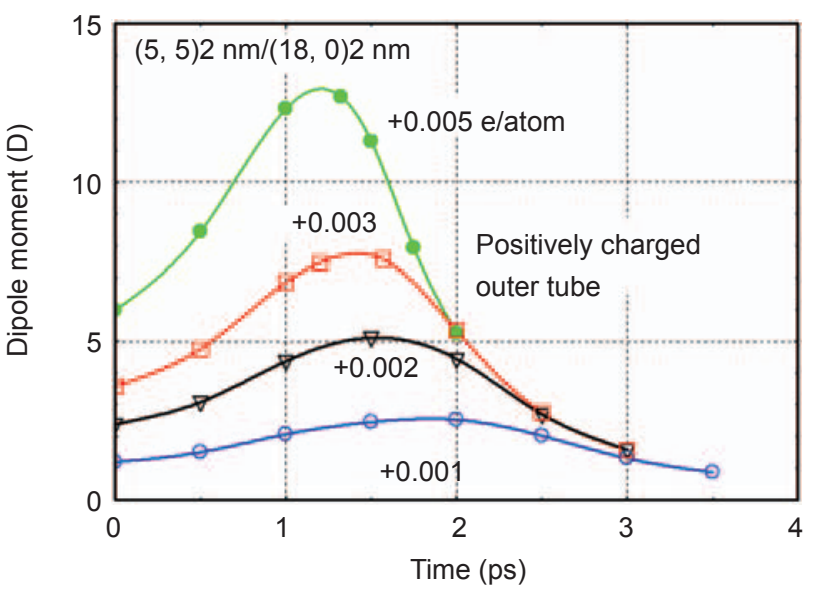

(b)

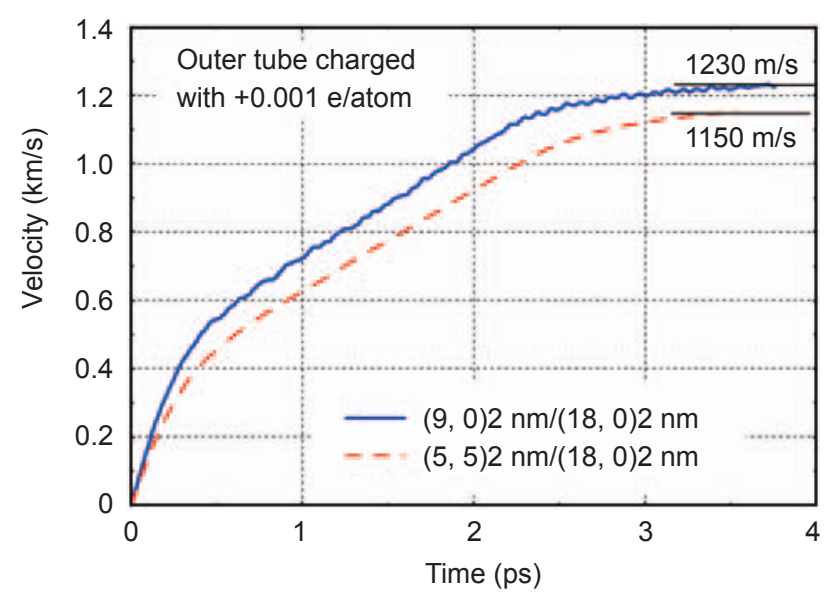

(c)

Figure 4 Influence of the charge density on the housing and the chirality of the core tube on the velocity and dipole moment of the neutral "bullets": (a) effect of the density of positive charge on the velocity of the "bullet"; (b) the corresponding dipole moment (in Debye units, D). Increasing the charge density on the housing shell leads to higher dipole moments and higher speeds of the "bullets"; (c) effect of chirality on the velocity of the "bullet" open end of the negatively charged housing creates a strong force field pushing the ball back inside it and also an absorbing force field in the vicinity of its open end. A fullerene ball put in front of the open end of a negatively charged housing can be absorbed inwards. The inset A in Fig. 5 shows that the ball can achieve inward acceleration in the vicinity of the entrance of the housing tube, except for a very small repulsive region where a slight outward acceleration exists. Therefore, a negative charge can be applied to the housing tube to refill "bullets". This absorbing-releasing cycle can serve as a novel nano manipulation technology. The different charge driving mechanisms operating at the nanoscale can be understood by the asymmetrical response of the electron orbitals to the charged housing environment. As shown in inset B in Fig. 5, when the $C_{60}$ moves along the housing tube, distinct distributions of its highest occupied molecular orbital (HOMO) can be found for the positively and negatively charged

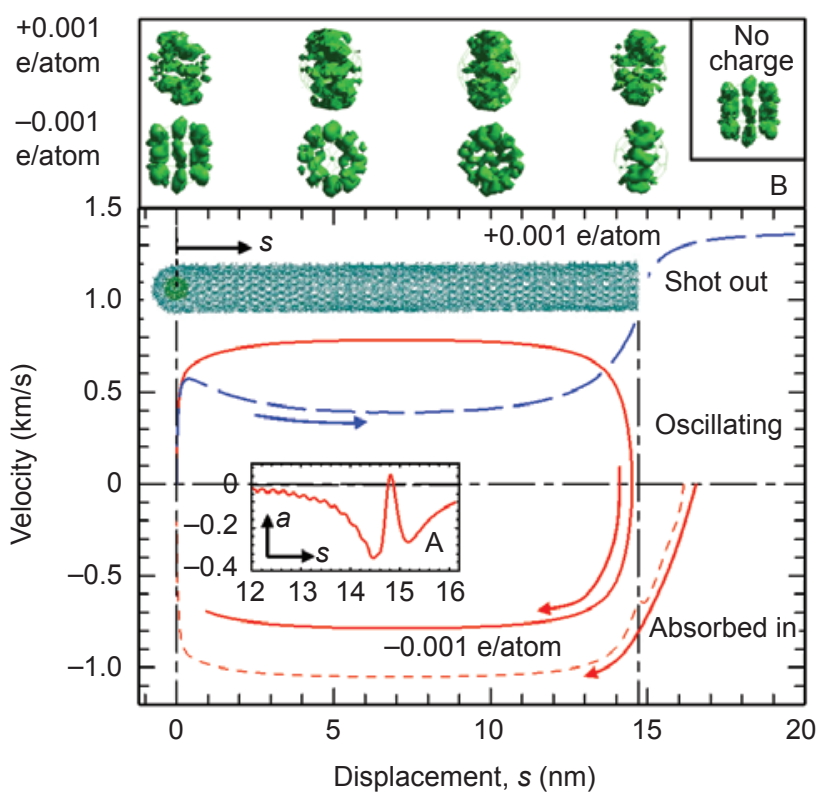

Figure 5 Movement of a $C_{60}$ ball in a long housing $(18,0)$ tube with positive or negative charge of density of 0.001 e/atom. A $C_{60}$ ball at the bottom (initial $S_{0}=0$ ) of a positively charged tube can be shot out as a "bullet" (blue), while a $C_{60}$ ball at the bottom or in front of the open end (initial $S_{0}=16.1 \mathrm{~nm}$ ) of a negatively charged tube can be driven into oscillation within the tube (red solid line) or absorbed into the tube (red dashed line). Inset $\mathrm{A}$ is the acceleration a $\left(\mathrm{nm} / \mathrm{ps}^{2}\right)$ of the absorbed $\mathrm{C}_{60}$ ball near the entrance of the open end of the housing tube. Inset B shows the variation in the density of the highest occupied molecular orbital ( $\mathrm{HOMO}$ ) of the $C_{60}$ when moving along the housing 
situations. This asymmetrical behaviour is responsible for the asymmetrical interaction force between the housing and the core part.

Controlling the charge of the system with an appropriate distribution in order to drive the core tube in a controllable way remains an intriguing technical issue. A conceivable way is electrostatic charging. When a static potential voltage is applied to the carbon nanotube, it will be electrically charged with the density proportional to the applied voltage [29]. Ion or electron beam irradiation may also be a possible way [30]. For example, after $\mathrm{Ga}^{+}$ion irradiation, the housing tube becomes positively charged [31].

\section{Conclusions}

In summary, based on QMMD simulations, we use an ideal model to show that a neutral $\mathrm{C}_{60}$ or CNT contained in an outer CNT can be driven into motion by arranging a charge distribution on the outer housing CNT. Positively charging the housing CNT with a uniform density of $0.001 \mathrm{e} /$ atom can shoot the neutral core out with speeds of over 1 $\mathrm{km} / \mathrm{s}$, like a "nanogun". Increasing the charge density can shoot the core out at higher speeds. A negatively charged housing tube can absorb an electrically neutral molecule in the vicinity of its open end and drive an encapsulated neutral core tube or ball into oscillation inside it, but cannot drive the core out. Thus the "nanogun" can be refilled or serve as a "nanomanipulator". This "nanogun" may have potential applications as drug or gene deliverers, switches, or nano-thrusters, and may open the door to conceptually new nano/molecular electromechanical devices.

\section{Acknowledgements}

The work is supported by the 973 Program (2007 CB936204), the Ministry of Education (No. 705021, IRT0534), and NSF (10732040) of China.

\section{References}

[1] Wang, X.; Song, J.; Liu, J.; Wang, Z. L. Direct-current nanogenerator driven by ultrasonic waves. Science 2007, 316,102-105.

[2] de Jonge, N.; Lamy, Y.; Schoots, K.; Oosterkamp, T. $\mathrm{H}$. High brightness electron beam from a multi-walled carbon nanotube. Nature 2002, 420, 393-395.

[3] Moseler, M.; Landman, U. Formation, stability, and breakup of nanojets. Science 2000, 289, 1165-1169.

[4] Fennimore, A. M.; Yuzvinsky, T. D.; Han, W. Q.; Fuhrer, M. S.; Cumings, J.; Zettl, A. Rotational actuators based on carbon nanotubes. Nature 2003, 424, 408-410.

[5] Baughman, R. H.; Cui, C.; Zakhidov, A. A.; Iqbal, Z.; Barisci, J. N.; Spinks, G. M.; Wallace, G. G.; Mazzoldi, A.; De Rossi, D.; Rinzler, A. G.; Jaschinski, O.; Roth, S.; Kertesz, M. Carbon nanotube actuators. Science 1999, 284, 1340-1344.

[6] Siwy, Z.; Fulinski, A. Fabrication of a synthetic nanopore ion pump. Phys. Rev. Lett. 2002, 89, 198103.

[7] Léger, Y.; Besombes, L.; Fernández-Rossier, J.; Maingault, L.; Mariette, H. Electrical control of a single $\mathrm{Mn}$ atom in a quantum dot. Phys. Rev. Lett. 2006, 97, 107401.

[8] Ahn, C. H. K.; Rabe, M.; Triscone, J.-M. Ferroelectricity at the nanoscale: Local polarization in oxide thin films and heterostructures. Science 2004, 303,488-491.

[9] Gong, X.; Li, J. Lu, H.; Wan, R.; Li, J.; Hu, J.; Fang, H. A charge-driven molecular water pump. Nat. Nanotechnol. 2007, 2, 709-712.

[10] Hinds, B. Molecular dynamics: A blueprint for a nanoscale pump. Nat. Nanotechnol. 2007, 2, 673-674.

[11] Yoshida, M.; Muneyuki, E.; Hisabori, T. ATP synthase A marvellous rotary engine of the cell. Nat. Rev. Mol. Cell Bio. 2001, 2, 669-677.

[12] Soong, R. K.; Bachand, G. D.; Neves, H. P.; Olkhovets, A. G.; Craighead, H. G. Montemagno, C. D. Powering an inorganic nanodevice with a biomolecular motor. Science 2000, 290, 1555-1558.

[13] Ahern, C. A.; Horn, R. Stirring up controversy with a voltage sensor paddle. Trends Neurosci. 2004, 27, 303307.

[14] Ishii, D.; Kinbara, K.; Ishida, Y.; Ishii, N.; Okochi, M.; Yohda, M.; Aida, T. Chaperonin-mediated stabilization and ATP-triggered release of semiconductor nanoparticles. Nature 2003, 423, 628-632.

[15] Sigworth, F. J. Structural biology: Life's transistors. Nature 2003, 423, 21-22.

[16] Perozo, E.; Rees, D. C. Structure and mechanism in prokaryotic mechanosensitive channels. Curr. Opin. Struc. 
Biol. 2003, 13, 432-442.

[17] Service, R. F. Superstrong nanotubes show they are smart, too. Science 1998, 281, 940-942.

[18] Chen, R. J.; Bangsaruntip, S.; Drouvalakis, K. A.; Kam, N. W. S.; Shim, M.; Li, Y.; Kim, W.; Utz, P. J.; Dai, H. Noncovalent functionalization of carbon nanotubes for highly specific electronic biosensors. P. Natl. Acad. Sci. USA 2003, 100, 4984-4989.

[19] Guo, W.; Guo, Y. Giant axial electrostrictive deformation in carbon nanotubes. Phys. Rev. Lett. 2003, 91, 115501.

[20] Lee, J.; Kim, H.; Kahng, S.-J.; Kim, G.; Son,Y.-W.; Ihm, J.; Kato, H.; Wang, Z. W.; Okazaki, T.; Shinohara, H.; Kuk, Y. Bandgap modulation of carbon nanotubes by encapsulated metallofullerenes. Nature 2002, 415, 10051008.

[21] Kwon, Y. K.; Tománek, D. lijima, S. "Bucky shuttle" memory device: Synthetic approach and molecular dynamics simulations. Phys. Rev. Lett. 1999, 82, 14701473.

[22] Cumings, J.; Zettl, A. Low-friction nanoscale linear bearing realized from multiwall carbon nanotubes. Science 2000, 289, 602-604.

[23] Guo, W.; Guo, Y.; Gao, H.; Zheng, Q.; Zhong, W. Energy dissipation in gigahertz oscillators from multiwalled carbon nanotubes. Phys. Rev. Lett. 2003, 91, 125501.

[24] Cummings, J.; Zettl, A. Localization and nonlinear resistance in telescopically extended nanotubes. Phys. Rev. Lett. 2004, 93, 086801.

[25] Rydberg, H.; Dion, M.; Jacobson, N.; Schröder, E.; Hyldgaard, P.; Simak, S. I.; Langreth, D. C.; Lundqvist, B. I. Van der Waals density functional for layered structures. Phys. Rev. Lett. 2003, 91, 126402.

[26] Stewart, J. J. P. Optimization of parameters for semiempirical methods I. Method. J. Comput. Chem. 1989, 10, 209-220.

[27] Stewart, J. J. P. Optimization of parameters for semiempirical methods II. applications. J. Comput. Chem. 1989, 10, 221-264.

[28] Dion, M.; Rydberg, H.; Schröder, E.; Langreth, D. C.; Lundqvist, B. I. Van der Waals density functional for general geometries. Phys. Rev. Lett. 2004, 92, 246401.

[29] Poncharal, P.; Wang, Z. L.; Ugarte, D.; Heer, W. A. Electrostatic deflections and electromechanical resonances of carbon nanotubes. Science 1999, 283, 1513-1516.

[30] Keblinski, P.; Nayak, S. K.; Zapol, P.; Ajayan, P. M. Charge distribution and stability of charged carbon nanotubes. Phys. Rev. Lett. 2002, 89, 255503.

[31] Wei, B. Q.; D'Arcy-Gall, J.; Ajayan, P. M.; Ramanath, G. Tailoring structure and electrical properties of carbon nanotubes using kilo-electron-volt ions. Appl. Phys. Lett. 2003, 83, 3851-3853. 